\title{
A FRACTAL OPERATOR ON SOME STANDARD SPACES OF FUNCTIONS
}

\author{
P. VISWANATHAN ${ }^{1}$ AND M. A. NAVASCUÉS ${ }^{2}$ \\ ${ }^{1}$ Mathematical Sciences Institute, Australian National University, \\ Canberra, ACT 200, Australia \\ ${ }^{2}$ Departmento de Matemática Aplicada, Escuela de Ingeniería y Arquitectura, \\ Universidad de Zaragoza, C/ María de Luna 3, Zaragoza 50018, Spain
}

(Received 19 May 2015)
Email addres needed for corresponding author. Also, be 'ACT 2601' and is the first is the first intiation full postal address?

\begin{abstract}
Through appropriate choices of elements in the underlying iterated function system, the methodology of fractal interpolation enables us to associate a family of continuous self-referential functions with a prescribed real-valued continuous function on a real compact interval. This procedure elicits what is referred to as an $\alpha$-fractal operator on $\mathcal{C}(I)$, the space of all real-valued continuous functions defined on a compact interval $I$. With an eye towards connecting fractal functions with other branches of mathematics, in this paper we continue to investigate the fractal operator in more general spaces such as the space $\mathcal{B}(I)$ of all bounded functions and the Lebesgue space $\mathcal{L}^{p}(I)$, and in some standard spaces of smooth functions such as the space $\mathcal{C}^{k}(I)$ of $k$-times continuously differentiable functions, Hölder spaces $\mathcal{C}^{k, \sigma}(I)$ and Sobolev spaces $\mathcal{W}^{k, p}(I)$. Using properties of the $\alpha$-fractal operator, the existence of Schauder bases consisting of self-referential functions for these function spaces is established.
\end{abstract}

Keywords: fractal functions; fractal operator; function spaces; topological isomorphism; Schauder basis 2010 Mathematics subject classification: Primary 28A80; 47A05; 47H09; 58C05; 65D05

\section{Introduction}

It is well known that the fractal interpolation function (FIF), a notion introduced by Barnsley $[\mathbf{1}, \mathbf{2}]$, provides an effective tool for the approximation of rough functions and an alternative to the traditional nonrecursive approximation methods that generally use smooth functions. The fractal function was originally introduced as a continuous function interpolating a prescribed set of data points in the Cartesian plane. These fractal approximants are constructed as attractors of appropriate iterated function systems (IFSs). Furthermore, a fractal function is the fixed point of a suitable contraction map (a ReadBajraktarević operator) defined on a subset of the space of all continuous real-valued functions (see $\S 2$ ).

Besides being a source of smooth and non-smooth approximants catering to the modelling problem at hand, the notion of FIF provides a bounded linear operator, termed an $\alpha$-fractal operator, on the space $\mathcal{C}(I)$ of all continuous real-valued functions on a compact interval $I$ in $\mathbb{R}$, the set of reals. In earlier papers [14-17], Navascués studied this fractal
Your paper has been reasonably heavily edited and carefully to ensure that no changes that distort your intended meaning have been made. Bear in mind, have misunderstood your original sentence, then reverting to your likely to confuse a least some readers so please consider clarification where possible.

I have presumed all subject classifications are primary - OK? O should some be secondary? should be checked though, that if I 
operator in detail. This operator-theoretic formalism of fractal functions enables them to interact with other traditional branches of pure and applied mathematics including functional analysis, operator theory, complex analysis, harmonic analysis and approximation theory.

In this paper we continue to explore the aforementioned fractal operator, but now within the setting of function spaces such as the space of bounded functions $\mathcal{B}(I)$, Lebesgue spaces $\mathcal{L}^{p}(I)$, the space of $k$-times continuously differentiable functions $\mathcal{C}^{k}(I)$, Hölder spaces $\mathcal{C}^{k, \sigma}(I)$ and Sobolev spaces $\mathcal{W}^{k, p}(I)$, all of which are prevalent in modern analysis and function theory. Hence, it is our view that the present work enriches the theory of fractal functions and opens the door to them finding further applications in various fields such as numerical analysis, functional analysis and harmonic analysis (in connection with partial differential equations, for example). In particular, we expect that the current study will pave the way for investigating shape-preserving fractal approximation in the various function spaces considered herein. For shape-preserving fractal approximation in the space of continuous functions, the reader is invited to refer to [22].

The research reported here is admittedly influenced to an extent by works by Massopust on fractal functions and local fractal functions: see [9-13]. However, it should be noted that our exposition has a different goal. Our main observations are centred on the $\alpha$-fractal operator defined on some standard spaces of functions, in contrast to the Read-Bajraktarević operator that defines fractal functions, and we find this link quite intriguing.

Turning to the structure of our paper, in $\S 2$ we assemble the requisite general material. In $\S 3$ we revisit the construction of $\alpha$-fractal functions in various spaces of functions, and this acts as a prelude to our main findings, which are given in the last section. The last section, $\S 4$, concerns the development of some foundational aspects of the associated fractal operator.

\section{Notation and preliminaries}

In this section we provide a brief introduction to various spaces of functions that we discuss in this paper. We them review the requisite background material on fractal functions. We recommend that interested readers consult $[\mathbf{1}, \mathbf{1 4}, \mathbf{2 0}, \mathbf{2 1}]$ for further details.

The set of natural numbers will be denoted by $\mathbb{N}$ and the set of real numbers by $\mathbb{R}$. For a fixed $N \in \mathbb{N}$, we shall write $\mathbb{N}_{N}$ for the set of the first $N$ natural numbers. By a self-map we mean a function whose domain and codomain are the same. For a given compact interval $I=[c, d] \subset \mathbb{R}$, let

$$
\mathcal{B}(I):=\{g: I \rightarrow \mathbb{R} ; g \text { is bounded on } I\} .
$$

The functional

$$
\|g\|_{\infty}:=\sup \{|g(x)|: x \in I\}
$$

termed the uniform norm, turns $\mathcal{B}(I)$ into a Banach space. For $k \in \mathbb{N} \cup\{0\}$, consider the space

$$
\mathcal{C}^{k}(I):=\left\{g: I \rightarrow \mathbb{R} ; g \text { is } k \text {-times differentiable and } g^{(k)} \in \mathcal{C}(I)\right\}
$$


endowed with the norm

$$
\|g\|_{\mathcal{C}^{k}}:=\max \left\{\left\|g^{(r)}\right\|_{\infty}: r \in \mathbb{N}_{k} \cup\{0\}\right\} .
$$

In addition to the Banach spaces $\mathcal{B}(I)$ and $\mathcal{C}^{k}(I)$, we require the following spaces that have numerous applications in analysis.

For $0<p \leqslant \infty$, let

$$
\mathcal{L}^{p}(I):=\left\{g: I \rightarrow \mathbb{R} ; g \text { is measurable and }\|g\|_{p}<\infty\right\},
$$

where the 'norm' is given by

$$
\|g\|_{p}= \begin{cases}{\left[\int_{I}|g(x)|^{p} \mathrm{~d} x\right]^{1 / p},} & 0<p<\infty, \\ \underset{x \in I}{\operatorname{ess} \sup }|g(x)|, & p=\infty .\end{cases}
$$

We recall that for $1 \leqslant p \leqslant \infty,\|\cdot\|_{p}$ defines a norm on $\mathcal{L}^{p}(I)$, and $\left(\mathcal{L}^{p}(I),\|\cdot\|_{p}\right)$ is a Banach space. Note that for $p=2$, the space $\mathcal{L}^{2}(I)$ is a Hilbert space with respect to the inner product

$$
\left\langle g_{1}, g_{2}\right\rangle:=\int_{I} g_{1} g_{2} \mathrm{~d} x .
$$

For $0<p<1,\|\cdot\|_{p}$ is not really a norm, only a quasi-norm, that is, in place of the triangle inequality we have

$$
\left\|g_{1}+g_{2}\right\|_{p} \leqslant 2^{1 / p}\left(\left\|g_{1}\right\|_{p}+\left\|g_{2}\right\|_{p}\right)
$$

and $\mathcal{L}^{p}(I)$ is a quasi-Banach space.

Let $u, v \in \mathcal{L}^{1}(I)$. We say that $v$ is the $k$ th weak derivative of $u$ and write $\mathrm{D}^{k} u=v$, provided

$$
\int_{I} u \mathrm{D}^{k} \varphi \mathrm{d} x=(-1)^{k} \int_{I} v \varphi \mathrm{d} x
$$

for all infinitely differentiable functions $\varphi$ with $\varphi(c)=\varphi(d)=0$.

We usually use upright ' $\mathrm{D}$ ' for derivatives (and
always use 'd' for the differential), hence the changes here. Please mark any ' $D$ ' that should be ' $D$ ' and vice versa.

For $1 \leqslant p \leqslant \infty$ and $k \in \mathbb{N} \cup\{0\}$, let $\mathcal{W}^{k, p}(I)$ denote the usual Sobolev space. That is,

$$
\mathcal{W}^{k, p}(I):=\left\{g: I \rightarrow \mathbb{R} ; \mathrm{D}^{j} g \in \mathcal{L}^{p}(I), j=0,1, \ldots, k\right\},
$$

where $\mathrm{D}^{j} g$ denotes the $j$ th weak or distributional derivative of $g$. The space $\mathcal{W}^{k, p}(I)$ endowed with the norm

$$
\|g\|_{\mathcal{W}^{k, p}}:= \begin{cases}{\left[\sum_{j=0}^{k}\left\|\mathrm{D}^{j} g\right\|_{p}^{p}\right]^{1 / p},} & \text { for } p \in[1, \infty), \\ \sum_{j=0}^{k}\left\|\mathrm{D}^{j} g\right\|_{\infty}, & \text { for } p=\infty\end{cases}
$$


is a Banach space. For $p=2$, it is customary to denote $\mathcal{W}^{k, p}(I)$ by $\mathcal{H}^{k}(I)$, which is a Hilbert space.

A function $u: I \rightarrow \mathbb{R}$ is said to be Hölder continuous with exponent $\sigma$ if

$$
|u(x)-u(y)| \leqslant C|x-y|^{\sigma} \quad \text { for all } x, y \in I \text { and for some } C>0 .
$$

For Hölder continuous functions $u$ with exponent $\sigma$, let us define the $\sigma$ th Hölder seminorm as

$$
[u]_{\sigma}=\sup _{x, y \in I, x \neq y} \frac{|u(x)-u(y)|}{|x-y|^{\sigma}}
$$

and consider the Hölder space

$$
\mathcal{C}^{k, \sigma}(I):=\left\{g \in \mathcal{C}^{k-1}(I) ; g^{(k)} \text { is Hölder continuous with exponent } \sigma\right\} .
$$

The space $\mathcal{C}^{k, \sigma}(I)$ is a Banach space when endowed with the norm

$$
\|g\|_{\mathcal{C}^{k, \sigma}}:=\sum_{j=0}^{k}\left\|g^{(j)}\right\|_{\infty}+\left[g^{(k)}\right]_{\sigma} .
$$

Having exposed the reader to function spaces that we will encounter in due course, next we provide an overview of fractal interpolation and related ideas. Assume that $N \in \mathbb{N}, N>2$. Let $\left\{\left(x_{i}, y_{i}\right) \in \mathbb{R}^{2}: i \in \mathbb{N}_{N}\right\}$ denote the prescribed set of interpolation data with strictly increasing abscissae. Set $I=\left[x_{1}, x_{N}\right]$ and $I_{i}=\left[x_{i}, x_{i+1}\right]$ for $i \in \mathbb{N}_{N-1}$. Fractal interpolation constructs a continuous function $g: I \rightarrow \mathbb{R}$ satisfying $g\left(x_{i}\right)=y_{i}$ for all $i \in \mathbb{N}_{N}$ and whose graph $G(g)$ is a fractal in the sense that $G(g)$ is a union of transformed copies of itself (see (2.1)). Suppose that $L_{i}: I \rightarrow I_{i}, i \in \mathbb{N}_{N-1}$, are affinities satisfying

$$
L_{i}\left(x_{1}\right)=x_{i}, \quad L_{i}\left(x_{N}\right)=x_{i+1} .
$$

For $i \in \mathbb{N}_{N-1}$, let $F_{i}: I \times \mathbb{R} \rightarrow \mathbb{R}$ be continuous functions fulfilling

$$
\left|F_{i}(x, y)-F_{i}\left(x, y^{*}\right)\right| \leqslant c_{i}\left|y-y^{*}\right|, \quad F_{i}\left(x_{1}, y_{1}\right)=y_{i}, \quad F_{i}\left(x_{N}, y_{N}\right)=y_{i+1}
$$

for $y, y^{*} \in \mathbb{R}$ and $0<c_{i}<1$. Define

$$
\mathcal{C}_{y_{1}, y_{N}}(I)=\left\{h \in \mathcal{C}(I) ; h\left(x_{1}\right)=y_{1}, h\left(x_{N}\right)=y_{N}\right\} .
$$

It is readily observed that $\mathcal{C}_{y_{1}, y_{N}}(I)$ is a closed (metric) subspace of the Banach space $\left(\mathcal{C}(I),\|\cdot\|_{\infty}\right)$. Define the Read-Bajraktarević (RB) operator $T: \mathcal{C}_{y_{1}, y_{N}}(I) \rightarrow \mathcal{C}_{y_{1}, y_{N}}(I)$ via

$$
(T h)(x)=F_{i}\left(L_{i}^{-1}(x), h \circ L_{i}^{-1}(x)\right), \quad x \in I_{i}, i \in \mathbb{N}_{N-1} .
$$

The nonlinear mapping $T$ is a contraction with a contraction factor $c:=\max \left\{c_{i}: i \in\right.$ $\mathbb{N}_{N-1}$ \}. Consequently, by the Banach fixed-point theorem, $T$ has a unique fixed point, say $g$. Furthermore, $g$ interpolates the data $\left\{\left(x_{i}, y_{i}\right): i \in \mathbb{N}_{N}\right\}$ and enjoys the functional equation

$$
g\left(L_{i}(x)\right)=F_{i}(x, g(x)), \quad x \in I, i \in \mathbb{N}_{N-1} .
$$

What do you mean by 'enjoys' here? Clarification possible? 
Let $w_{i}: I \times \mathbb{R} \rightarrow I_{i} \times \mathbb{R} \subset I \times \mathbb{R}$ be defined by

$$
w_{i}(x, y)=\left(L_{i}(x), F_{i}(x, y)\right), \quad i \in \mathbb{N}_{N-1} .
$$

Consider the IFS $W=\left\{I \times \mathbb{R} ; w_{i}, i \in \mathbb{N}_{N-1}\right\}$. The attractor $\mathrm{G}$ of the IFS $W$ is then the graph $G(g)$ of the function $g$, that is,

$$
G(g)=\bigcup_{i \in \mathbb{N}_{N-1}} w_{i}(G(g)) .
$$

Therefore, $g$ is a self-referential function.

Navascués [14] observed that the theory of FIF can be used to generate a family of continuous functions having fractal characteristics from a prescribed continuous function. To this end, for a given $f \in \mathcal{C}(I)$, consider a partition $\Delta:=\left\{x_{1}, x_{2}, \ldots, x_{N}\right\}$ of $I$ satisfying $x_{1}<x_{2}<\cdots<x_{N}$, a continuous map $b: I \rightarrow \mathbb{R}$ such that

$$
b \neq f, \quad b\left(x_{1}\right)=f\left(x_{1}\right), \quad b\left(x_{N}\right)=f\left(x_{N}\right),
$$

and $N-1$ real numbers $\alpha_{i}$ satisfying $\left|\alpha_{i}\right|<1$. Define an IFS through the maps

$$
L_{i}(x)=a_{i} x+d_{i}, \quad F_{i}(x, y)=\alpha_{i} y+f \circ L_{i}(x)-\alpha_{i} b(x), \quad i \in \mathbb{N}_{N-1} .
$$

The corresponding FIF, denoted by $f_{\Delta, b}^{\alpha}=f^{\alpha}$, is referred to as an $\alpha$-fractal function for $f$ (a fractal perturbation of $f$ ) with respect to a scale vector $\alpha=\left(\alpha_{1}, \alpha_{2}, \ldots, \alpha_{N-1}\right)$, base function $b$ and partition $\Delta$. Here, the interpolation points are $\left\{\left(x_{i}, f\left(x_{i}\right)\right): i \in \mathbb{N}_{N}\right\}$. The fractal dimension (Minkowski dimension or Hausdorff dimension) of the function $f^{\alpha}$ depends on the parameter $\alpha \in(-1,1)^{N-1}$. The function $f^{\alpha}$ is the fixed point of the operator $T_{\Delta, b, f}^{\alpha}: \mathcal{C}_{f}(I) \rightarrow \mathcal{C}_{f}(I)$ defined by

$$
\left(T_{\Delta, b, f}^{\alpha} g\right)(x)=f(x)+\alpha_{i}(g-b) \circ L_{i}^{-1}(x), \quad x \in I_{i}, i \in \mathbb{N}_{N-1},
$$

where $\mathcal{C}_{f}(I):=\left\{g \in \mathcal{C}(I) ; g\left(x_{1}\right)=f\left(x_{1}\right), g\left(x_{N}\right)=f\left(x_{N}\right)\right\}$. Consequently, the $\alpha$-fractal function corresponding to $f$ satisfies the self-referential equation

$$
f_{\Delta, b}^{\alpha}(x)=f(x)+\alpha_{i}\left(f_{\Delta, b}^{\alpha}-b\right)\left(L_{i}^{-1}(x)\right), \quad x \in I_{i}, i \in \mathbb{N}_{N-1} .
$$

Assume that the base function $b$ depends linearly on $f$, say $b=L f$, where $L: \mathcal{C}(I) \rightarrow \mathcal{C}(I)$ is a bounded linear map. Then the map

Changes to

$$
\mathcal{F}_{\Delta, b}^{\alpha}: \mathcal{C}(I) \rightarrow \mathcal{C}(I), \quad \mathcal{F}_{\Delta, b}^{\alpha}(f)=f_{\Delta, b}^{\alpha}
$$

which is referred to as an $\alpha$-fractal operator, is a bounded linear operator.

To obtain fractal functions with more flexibility, iterated function systems in which scaling factors are replaced by scaling functions have received attention in the recent fractal functions literature $[\mathbf{3}, \mathbf{4}, \mathbf{7}, \mathbf{1 2}]$. That is, one may consider the IFS with maps

$$
L_{i}(x)=a_{i} x+d_{i}, \quad F_{i}(x, y)=\alpha_{i}(x) y+f \circ L_{i}(x)-\alpha_{i}(x) b(x), \quad i \in \mathbb{N}_{N-1},
$$


where the $\alpha_{i}$ are continuous functions satisfying $\max \left\{\left\|\alpha_{i}\right\|_{\infty}: i \in \mathbb{N}_{N-1}\right\}<1$, and $b \neq f$ is a continuous function that agrees with $f$ at the extremes of the interval $I$ (see, for example, [25]). The corresponding $\alpha$-fractal function is the fixed point of the RB operator

$$
\left(T_{\Delta, b, f}^{\alpha} g\right)(x)=f(x)+\alpha_{i}\left(L_{i}^{-1}(x)\right)(g-b)\left(L_{i}^{-1}(x)\right), \quad x \in I_{i}, \quad i \in \mathbb{N}_{N-1},
$$

and hence obeys the functional equation

$$
f_{\Delta, b}^{\alpha}(x)=f(x)+\alpha_{i}\left(L_{i}^{-1}(x)\right)\left(f_{\Delta, b}^{\alpha}-b\right)\left(L_{i}^{-1}(x)\right), \quad x \in I_{i}, i \in \mathbb{N}_{N-1} .
$$

\section{3. $\alpha$-fractal functions on various function spaces}

The aim of this section is to equip the reader with constructions of $\alpha$-fractal functions in various function spaces, and as such it forms a prelude to our main results in the next section. The said constructions can be obtained as particular cases of the constructions of fractal functions and local fractal functions in the corresponding function spaces reported in various places in the literature $[\mathbf{9 - 1 3}]$. We will present the constructions in the form of various theorems, the proofs of which can be found in the references just given unless indicated otherwise.

Theorem 3.1. Let $f \in \mathcal{B}(I)$. Assume that $\Delta:=\left\{x_{1}, x_{2}, \ldots, x_{N}\right\}$ is a partition of $I$ with strictly increasing abscissae, and let $I_{i}=\left[x_{i}, x_{i+1}\right)$, for $i \in \mathbb{N}_{N-2}$, and $I_{N-1}=$ $\left[x_{N-1}, x_{N}\right]$ be the corresponding subintervals. Further assume that the maps $L_{i}: I \rightarrow I_{i}$ are affinities satisfying $L_{i}\left(x_{1}\right)=x_{i}, L_{i}\left(x_{N}^{-}\right)=x_{i+1}$ for $i \in \mathbb{N}_{N-1}$, and the base function $b$ and scaling functions $\alpha_{i}, i \in \mathbb{N}_{N-1}$, are real-valued bounded functions on $I$. Then the $R B$ operator defined in (2.6) is a well-defined self-map on $\mathcal{B}(I)$. Furthermore, if $\|\alpha\|_{\infty}=\max \left\{\left\|\alpha_{i}\right\|_{\infty}: i \in \mathbb{N}_{N-1}\right\}<1$, then $T$ is a contraction and has a unique fixed point $f^{\alpha} \in \mathcal{B}(I)$.

Theorem 3.2. Let $f \in \mathcal{L}^{p}(I), 0<p \leqslant \infty$. Suppose that $\Delta=\left\{x_{1}, x_{2}, \ldots, x_{N}\right\}$ is a partition of $I$ satisfying $x_{1}<x_{2}<\cdots<x_{N}, I_{i}:=\left[x_{i}, x_{i+1}\right)$ for $i \in \mathbb{N}_{N-2}$, and $I_{N-1}:=\left[x_{N-1}, x_{N}\right]$. Let $L_{i}: I \rightarrow I_{i}$ be affine maps $L_{i}(x)=a_{i} x+d_{i}$ satisfying $L_{i}\left(x_{1}\right)=x_{i}$ and $L_{i}\left(x_{N}^{-}\right)=x_{i+1}$ for $i \in \mathbb{N}_{N-1}$. Choose $\alpha_{i} \in \mathcal{L}^{\infty}(I)$ for all $i \in \mathbb{N}_{N-1}$ and $b \in \mathcal{L}^{p}(I)$. Then the $R B$ operator $T$ given in (2.6) defines a self-map on $\mathcal{L}^{p}(I)$. Furthermore, for the scaling functions $\alpha_{i}, i \in \mathbb{N}_{N-1}$, satisfying the following condition, $T$ is a contraction map on $\mathcal{L}^{p}(I)$ :

$$
\begin{aligned}
{\left[\sum_{i \in \mathbb{N}_{N-1}} a_{i}\left\|\alpha_{i}\right\|_{\infty}^{p}\right]^{1 / p}<1 } & \text { for } p \in[1, \infty) \\
\max _{i \in \mathbb{N}_{N-1}}\left\|\alpha_{i}\right\|_{\infty}<1 & \text { for } p=\infty \\
\sum_{i \in \mathbb{N}_{N-1}} a_{i}\left\|\alpha_{i}\right\|_{\infty}^{p}<1 & \text { for } p \in(0,1)
\end{aligned}
$$

Consequently, the corresponding fixed point $f^{\alpha} \in \mathcal{L}^{p}(I)$ obeys the self-referential equation (2.7). 
Recall that, in most cases, the $\alpha$-fractal function has non-integer Hausdorff and Minkowski dimensions that depend on the scale vector $\alpha$, and the map $f \mapsto f^{\alpha}$ is therefore a 'roughing' operation. The following theorem provides appropriate choices of the scaling functions and base function so that the order of continuity of $f$ can be preserved in $f^{\alpha}$ (see $\left.[?, 23]\right)$.

Theorem 3.3. Let $f \in \mathcal{C}^{k}(I)$, where $k \in \mathbb{N}$. Suppose that $\Delta=\left\{x_{1}, x_{2}, \ldots, x_{N}\right\}$ is a partition of $I$ satisfying $x_{1}<x_{2}<\cdots<x_{N}, I_{i}:=\left[x_{i}, x_{i+1}\right]$ for $i \in \mathbb{N}_{N-1}$, and $L_{i}: I \rightarrow I_{i}$ are affine maps $L_{i}(x)=a_{i} x+d_{i}$ satisfying $L_{i}\left(x_{1}\right)=x_{i}$ and $L_{i}\left(x_{N}\right)=x_{i+1}$ for $i \in \mathbb{N}_{N-1}$. Suppose that $k$-times continuously differentiable scaling functions and base function are selected so that

$$
\begin{gathered}
\left\|\alpha_{i}\right\|_{\mathcal{C}^{k}} \leqslant\left(\frac{a_{i}}{2}\right)^{k}, \\
b^{(r)}\left(x_{1}\right)=f^{(r)}\left(x_{1}\right), \quad b^{(r)}\left(x_{N}\right)=f^{(r)}\left(x_{N}\right), \quad i \in \mathbb{N}_{N-1}, r \in \mathbb{N}_{k} \cup\{0\} .
\end{gathered}
$$

The $R B$ operator defined in (2.6) is then a contraction on the complete metric space

$$
\mathcal{C}_{f}^{k}(I):=\left\{g \in \mathcal{C}^{k}(I) ; g^{(r)}\left(x_{1}\right)=f^{(r)}\left(x_{1}\right), g^{(r)}\left(x_{N}\right)=f^{(r)}\left(x_{N}\right), r \in \mathbb{N}_{k} \cup\{0\}\right\} .
$$

Furthermore, the derivative $\left(f^{\alpha}\right)^{(r)}$ of its unique fixed point $f^{\alpha}$ satisfies the self-referential equation

$$
\begin{aligned}
&\left(f^{\alpha}\right)^{(r)}(x)=f^{(r)}(x)+a_{i}^{-r}\left[\sum_{j=0}^{r}\left(\begin{array}{l}
r \\
j
\end{array}\right) \alpha_{i}^{(r-j)}\left(L_{i}^{-1}(x)\right)\left(f^{\alpha}-b\right)^{(j)}\left(L_{i}^{-1}(x)\right)\right] \\
& x \in I_{i}, i \in \mathbb{N}_{N-1},
\end{aligned}
$$

and, consequently, $f^{\alpha}$ agrees with $f$ at the knot points up to the $k$ th derivative.

To simplify the exposition, we assume a constant scaling function $\alpha_{i}(x)=\alpha_{i}$ for all

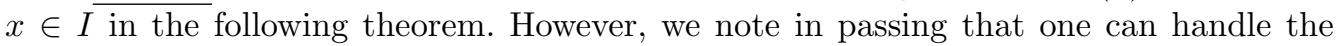
setting of scaling functions in a similar manner by using the following Leibnitz formula for weak derivatives $[\mathbf{6}]$.

Assume that $u \in \mathcal{W}^{k, p}(I)$ and that $\varphi$ is infinitely differentiable. Then $\varphi u \in \mathcal{W}^{k, p}(I)$ and for $j \leqslant k$

$$
\mathrm{D}^{j}(\varphi u)=\sum_{r=0}^{j}\left(\begin{array}{l}
j \\
r
\end{array}\right) \mathrm{D}^{r} \varphi \mathrm{D}^{j-r} u .
$$

Theorem 3.4. Let $f \in \mathcal{W}^{k, p}(I)$. Suppose that $\Delta=\left\{x_{1}, x_{2}, \ldots, x_{N}\right\}$ is a partition of $I$ with increasing abscissae, $I_{i}:=\left[x_{i}, x_{i+1}\right)$ for $i \in \mathbb{N}_{N-2}$, and $I_{N-1}:=\left[x_{N-1}, x_{N}\right]$. Let $L_{i}: I \rightarrow I_{i}$ be affine maps $L_{i}(x)=a_{i} x+d_{i}$ satisfying $L_{i}\left(x_{1}\right)=x_{i}$ and $L_{i}\left(x_{N}^{-}\right)=x_{i+1}$ for $i \in \mathbb{N}_{N-1}$. Assume that $b \in \mathcal{W}^{k, p}(I)$ and the scaling factors satisfy

$$
\begin{gathered}
{\left[\sum_{i \in \mathbb{N}_{N-1}} \frac{\left|\alpha_{i}\right|^{p}}{a_{i}^{k p-1}}\right]^{1 / p}<1 \quad \text { for } p \in[1, \infty),} \\
\max _{i \in \mathbb{N}_{N-1}}\left\{\frac{\left|\alpha_{i}\right|}{a_{i}^{k}}\right\}<1 \quad \text { for } p=\infty .
\end{gathered}
$$

One of the citations here points to a non-existent reference: do you mean $[23,24]$ ? 
The $R B$ operator (2.3) is then a contraction map on $\mathcal{W}^{k, p}(I)$, and the unique fixed point $f^{\alpha} \in \mathcal{W}^{k, p}(I)$ obeys the self-referential equation (??).

Theorem 3.5. Let $f \in \mathcal{C}^{k, \sigma}(I)$. Suppose that $\Delta=\left\{x_{1}, x_{2}, \ldots, x_{N}\right\}$ is a partition of $I$ satisfying $x_{1}<x_{2}<\cdots<x_{N}, I_{i}:=\left[x_{i}, x_{i+1}\right]$ for $i \in \mathbb{N}_{N-1}$, and $L_{i}: I \rightarrow I_{i}$ are affine maps $L_{i}(x)=a_{i} x+d_{i}$ satisfying $L_{i}\left(x_{1}\right)=x_{i}$ and $L_{i}\left(x_{N}\right)=x_{i+1}$ for $i \in \mathbb{N}_{N-1}$. Let the base function $b \in \mathcal{C}^{k, \sigma}(I)$ and the scaling factors $\alpha_{i}$ satisfy

$$
\begin{gathered}
\max \left\{\frac{\left|\alpha_{i}\right|}{a_{i}^{\sigma+k}}: i \in \mathbb{N}_{N-1}\right\}<1, \\
b^{(r)}\left(x_{1}\right)=f^{(r)}\left(x_{1}\right), \quad b^{(r)}\left(x_{N}\right)=f^{(r)}\left(x_{N}\right), \quad r \in \mathbb{N}_{k} \cup\{0\} .
\end{gathered}
$$

The $R B$ operator $T$ defined in (2.3) is then a contraction map on $\mathcal{C}_{f}^{k, \sigma}(I) \subset \mathcal{C}^{k, \sigma}(I)$ given by

$$
\mathcal{C}_{f}^{k, \sigma}(I):=\left\{g \in \mathcal{C}^{k, \sigma}(I) ; g^{(r)}\left(x_{1}\right)=f^{(r)}\left(x_{1}\right), g^{(r)}\left(x_{N}\right)=f^{(r)}\left(x_{N}\right), 0 \leqslant r \leqslant k\right\},
$$

and the unique fixed point $f^{\alpha} \in \mathcal{C}^{k, \sigma}(I)$ obeys the self-referential equation (??).

Proof. Following the proof of Theorem 3.3 we assert that $T g \in \mathcal{C}^{k}(I)$ whenever $g \in \mathcal{C}^{k, \sigma}(I)$. Furthermore,

There were two equations with the same label in your submission: should this say ' $(2.2)$ ' or '(2.4)'?

$$
(T g)^{(r)}(x)=f^{(r)}(x)+\frac{\alpha_{i}}{a_{i}^{r}}(g-b)^{(r)}\left(L_{i}^{-1}(x)\right), \quad x \in I_{i}, i \in \mathbb{N}_{N-1}, r \in \mathbb{N}_{k} \cup\{0\} .
$$

The $\sigma$ th Hölder seminorm of $(T g)^{(k)}$ is given by

$$
\begin{aligned}
{\left[(T g)^{(k)}\right]_{\sigma} } & =\sup _{x, y \in I, x \neq y} \frac{\left|(T g)^{(k)}(x)-(T g)^{(k)}(y)\right|}{|x-y|^{\sigma}} \\
& =\max _{i \in \mathbb{N}_{N-1}} \sup _{x, y \in I_{i}, x \neq y} \frac{\left|\left(\alpha_{i} / a_{i}^{k}\right)\left[(g-b)^{(k)}\left(L_{i}^{-1}(x)\right)-(g-b)^{(k)}\left(L_{i}^{-1}(y)\right)\right]\right|}{|x-y|^{\sigma}} \\
& \left.\leqslant \max _{i \in \mathbb{N}_{N-1}}\left(\frac{\left|\alpha_{i}\right|}{a_{i}^{k}}\right)_{x, y \in I_{i}, x \neq y} \sup _{\frac{\left|g^{(k)}\left(L_{i}^{-1}(x)\right)-g^{(k)}\left(L_{i}^{-1}(y)\right)\right|}{|x-y|^{\sigma}}}+\frac{\left|b^{(k)}\left(L_{i}^{-1}(x)\right)-b^{(k)}\left(L_{i}^{-1}(y)\right)\right|}{|x-y|^{\sigma}}\right] \\
& =\max _{i \in \mathbb{N}_{N-1}}\left(\frac{\left|\alpha_{i}\right|}{a_{i}^{k}}\right)_{x, y \in I_{i}, x \neq y} \frac{\left|g^{(k)}\left(L_{i}^{-1}(x)\right)-g^{(k)}\left(L_{i}^{-1}(y)\right)\right|}{a_{i}^{\sigma}\left|L_{i}^{-1}(x)-L_{i}^{-1}(y)\right|^{\sigma}} \\
& \left.+\frac{\left|b^{(k)}\left(L_{i}^{-1}(x)\right)-b^{(k)}\left(L_{i}^{-1}(y)\right)\right|}{a_{i}^{\sigma}\left|L_{i}^{-1}(x)-L_{i}^{-1}(y)\right|^{\sigma}}\right] \\
& =\max _{i \in \mathbb{N}_{N-1}}\left(\frac{\left|\alpha_{i}\right|}{a_{i}^{k+\sigma}}\right) \sup _{\tilde{x}, \tilde{y} \in I, \tilde{x} \neq \tilde{y}}\left[\frac{\left|g^{(k)}(\tilde{x})-g^{(k)}(\tilde{y})\right|}{|\tilde{x}-\tilde{y}|^{\sigma}}+\frac{\left|b^{(k)}(\tilde{x})-b^{(k)}(\tilde{y})\right|}{|\tilde{y}|^{\sigma}}\right] \\
& =\max _{i \in \mathbb{N}_{N-1}}\left(\frac{\left|\alpha_{i}\right|}{a_{i}^{k+\sigma}}\right)\left(\left[g^{(k)}\right]_{\sigma}+\left[b^{(k)}\right]_{\sigma}\right) .
\end{aligned}
$$

There were two equations with the same label in your submission: should '(2.4)'? 
Since $b$ and $g$ are in $\mathcal{C}^{k, \sigma}(I)$, the previous estimate ensures that $\left[(T g)^{(k)}\right]_{\sigma}<\infty$ and hence that $T g \in \mathcal{C}^{k, \sigma}(I)$. Again from (3.1), for $x \in I_{i}$,

$$
\begin{aligned}
\left|\left(T g_{1}-T g_{2}\right)^{(r)}(x)\right| & =\frac{\left|\alpha_{i}\right|}{a_{i}^{r}}\left|\left(g_{1}-g_{2}\right)^{(r)}\left(L_{i}^{-1}(x)\right)\right| \\
& \leqslant \frac{\left|\alpha_{i}\right|}{a_{i}^{r}}\left\|\left(g_{1}-g_{2}\right)^{(r)}\right\|_{\infty},
\end{aligned}
$$

and hence one readily obtains

$$
\left\|\left(T g_{1}-T g_{2}\right)^{(r)}\right\|_{\infty} \leqslant \max \left\{\frac{\left|\alpha_{i}\right|}{a_{i}^{r}}: i \in \mathbb{N}_{N-1}\right\}\left\|\left(g_{1}-g_{2}\right)^{(r)}\right\|_{\infty} .
$$

Along lines similar to the estimation of $\left[(T g)^{(k)}\right]_{\sigma}$, we get

$$
\left[\left(T g_{1}-T g_{2}\right)^{(k)}\right]_{\sigma} \leqslant \max \left\{\frac{\left|\alpha_{i}\right|}{a_{i}^{k+\sigma}}: i \in \mathbb{N}_{N-1}\right\}\left[\left(g_{1}-g_{2}\right)^{(k)}\right]_{\sigma} .
$$

From these computations we gather that

$$
\begin{aligned}
& \left\|T g_{1}-T g_{2}\right\|_{\mathcal{C}^{k, \sigma}} \\
& =\sum_{r=0}^{k}\left\|\left(T g_{1}-T g_{2}\right)^{(r)}\right\|_{\infty}+\left[\left(T g_{1}-T g_{2}\right)^{(k)}\right]_{\sigma} \\
& \leqslant \sum_{r=0}^{k} \max \left\{\frac{\left|\alpha_{i}\right|}{a_{i}^{r}}: i \in \mathbb{N}_{N-1}\right\}\left\|\left(g_{1}-g_{2}\right)^{(r)}\right\|_{\infty}+\max \left\{\frac{\left|\alpha_{i}\right|}{\left.a_{i}^{k+\sigma}: i \in \mathbb{N}_{N-1}\right\}\left[\left(g_{1}-g_{2}\right)^{(k)}\right]_{\sigma}}\right. \\
& \leqslant \max _{r \in \mathbb{N}_{k} \cup\{0\}} \max _{i \in \mathbb{N}_{N-1}}\left\{\frac{\left|\alpha_{i}\right|}{a_{i}^{r}}\right\} \sum_{r=0}^{k}\left\|\left(g_{1}-g_{2}\right)^{(r)}\right\|_{\infty}+\max _{i \in \mathbb{N}_{N-1}}\left\{\frac{\left|\alpha_{i}\right|}{a_{i}^{k+\sigma}}\right\}\left[\left(g_{1}-g_{2}\right)^{(k)}\right]_{\sigma} \\
& \leqslant \max \left\{\frac{\left|\alpha_{i}\right|}{a_{i}^{k+\sigma}}: i \in \mathbb{N}_{N-1}\right\}\left[\sum_{r=0}^{k}\left\|\left(g_{1}-g_{2}\right)^{(r)}\right\|_{\infty}+\left[\left(g_{1}-g_{2}\right)^{(k)}\right]_{\sigma}\right] \\
& =\max \left\{\frac{\left|\alpha_{i}\right|}{\left.a_{i}^{k+\sigma}: i \in \mathbb{N}_{N-1}\right\}\left\|g_{1}-g_{2}\right\|_{\mathcal{C}^{k, \sigma}} .}\right.
\end{aligned}
$$

The assumption on scaling factors now yields that $T$ is a contraction on $\mathcal{C}_{f}^{k, \sigma}(I)$, and the theorem is therefore proved.

\section{Fractal operators on function spaces}

Theorems 3.1-3.5 established in the previous section illustrate, albeit indirectly, the existence of an operator that assigns a function $f$ to its self-referential (fractal) analogue $f^{\alpha}$. To be precise, for a fixed set of scaling functions (factors) $\alpha_{i}$ and for a suitable choice of base function, there exists a map

$$
\mathcal{F}^{\alpha}: X \rightarrow X, \quad \mathcal{F}^{\alpha}(f)=f^{\alpha},
$$

Changes to sentence OK?

Change to 'operators' plural in heading OK? Or 'The fractal operator on...' or 'A fractal operator on...'?

Word added - OK? 
where $X$ is one of the Banach spaces $\mathcal{B}(I), \mathcal{C}^{k}(I), \mathcal{L}^{p}(I), \mathcal{W}^{k, p}(I)$ or $\mathcal{C}^{k, \sigma}(I)$. In this section we offer a couple of fundamental results on this fractal operator $\mathcal{F}^{\alpha}$. For definiteness, we shall work with $X=\mathcal{W}^{k, p}(I)$; other cases can be dealt with similarly. Furthermore, we assume that the base function $b$ depends on $f$ linearly and $b=L f$, where $L: X \rightarrow X$ is a linear operator that is bounded with respect to the norm in the corresponding space.

Proposition 4.1. For $f \in \mathcal{W}^{k, p}(I)$ and the scaling factors satisfying conditions prescribed in Theorem 3.4, we have

$$
\left\|f^{\alpha}-f\right\|_{\mathcal{W}^{k, p}} \leqslant \begin{cases}{\left[\sum_{i \in \mathbb{N}_{N-1}} \frac{\left|\alpha_{i}\right|^{p}}{a_{i}^{k p-1}}\right]^{1 / p}\left\|f^{\alpha}-b\right\|_{\mathcal{W}^{k, p}}} & \text { for } p \in[1, \infty), \\ \max \left\{\frac{\left|\alpha_{i}\right|}{a_{i}^{k}}: i \in \mathbb{N}_{N-1}\right\}\left\|f^{\alpha}-b\right\|_{\mathcal{W}^{k, p}} & \text { for } p=\infty\end{cases}
$$

Proof. We have the functional equation

$$
f^{\alpha}(x)=f(x)+\alpha_{i}\left(f^{\alpha}-b\right)\left(L_{i}^{-1}(x)\right), \quad x \in I_{i}, i \in \mathbb{N}_{N-1}
$$

Therefore,

$$
\left(\mathrm{D}^{k}\left(f^{\alpha}-f\right)\right)(x)=\frac{\alpha_{i}}{a_{i}^{k}}\left(\mathrm{D}^{k}\left(f^{\alpha}-b\right)\right)\left(L_{i}^{-1}(x)\right), \quad x \in I_{i}, \quad i \in \mathbb{N}_{N-1}
$$

Assume that $1 \leqslant p<\infty$. By a series of self-evident steps,

$$
\begin{aligned}
\left\|\mathrm{D}^{k}\left(f^{\alpha}-f\right)\right\|_{p}^{p} & =\int_{I}\left|\mathrm{D}^{k}\left(f^{\alpha}-f\right)(x)\right|^{p} \mathrm{~d} x \\
& =\sum_{i \in \mathbb{N}_{N-1}}\left(\frac{\left|\alpha_{i}\right|}{a_{i}^{k}}\right)^{p} \int_{I_{i}}\left|\mathrm{D}^{k}\left(f^{\alpha}-b\right)\left(L_{i}^{-1}(x)\right)\right|^{p} \mathrm{~d} x \\
& =\sum_{i \in \mathbb{N}_{N-1}}\left(\frac{\left|\alpha_{i}\right|}{a_{i}^{k}}\right)^{p} \int_{I} a_{i}\left|\mathrm{D}^{k}\left(f^{\alpha}-b\right)(\tilde{x})\right|^{p} \mathrm{~d} \tilde{x} \\
& =\sum_{i \in \mathbb{N}_{N-1}} \frac{\left|\alpha_{i}\right|^{p}}{a_{i}^{k p-1}}\left\|\mathrm{D}^{k}\left(f^{\alpha}-b\right)\right\|_{p}^{p}
\end{aligned}
$$

and, consequently,

$$
\left\|\mathrm{D}^{k}\left(f^{\alpha}-f\right)\right\|_{p}=\left[\sum_{i \in \mathbb{N}_{N-1}} \frac{\left|\alpha_{i}\right|^{p}}{a_{i}^{k p-1}}\right]^{1 / p}\left\|\mathrm{D}^{k}\left(f^{\alpha}-b\right)\right\|_{p}
$$


Thus,

$$
\begin{aligned}
\left\|f^{\alpha}-f\right\|_{\mathcal{W}^{k, p}} & =\left\|f^{\alpha}-f\right\|_{p}+\left\|\mathrm{D}^{k}\left(f^{\alpha}-f\right)\right\|_{p} \\
& =\left[\sum_{i \in \mathbb{N}_{N-1}} a_{i}\left|\alpha_{i}\right|^{p}\right]^{1 / p}\left\|f^{\alpha}-b\right\|_{p}+\left[\sum_{i \in \mathbb{N}_{N-1}} \frac{\left|\alpha_{i}\right|^{p}}{a_{i}^{k p-1}}\right]^{1 / p}\left\|\mathrm{D}^{k}\left(f^{\alpha}-b\right)\right\|_{p} \\
& \leqslant \max \left\{\left[\sum_{i \in \mathbb{N}_{N-1}} a_{i}\left|\alpha_{i}\right|^{p}\right]^{1 / p},\left[\sum_{i \in \mathbb{N}_{N-1}} \frac{\left|\alpha_{i}\right|^{p}}{a_{i}^{k p-1}}\right]^{1 / p}\right\}\left\|f^{\alpha}-b\right\|_{\mathcal{W}^{k, p}} \\
& =\left[\sum_{i \in \mathbb{N}_{N-1}} \frac{\left|\alpha_{i}\right|^{p}}{a_{i}^{k p-1}}\right]^{1 / p}\left\|f^{\alpha}-b\right\|_{\mathcal{W}^{k, p}} .
\end{aligned}
$$

The proof for $p=\infty$ is similar.

Theorem 4.2. Let $I_{d}$ be the identity operator on $\mathcal{W}^{k, p}(I), b=L f$, where $L$ is a bounded linear operator on $\mathcal{W}^{k, p}(I)$, and the scaling factors satisfy conditions prescribed in Theorem 3.4. Then

$$
\begin{array}{ll}
\left\|f^{\alpha}-f\right\|_{\mathcal{W}^{k, p}} \leqslant \frac{\left[\sum_{i \in \mathbb{N}_{N-1}}\left|\alpha_{i}\right|^{p} / a_{i}^{k p-1}\right]^{1 / p}}{1-\left[\sum_{i \in \mathbb{N}_{N-1}}\left|\alpha_{i}\right|^{p} / a_{i}^{k p-1}\right]^{1 / p}}\left\|I_{d}-L\right\|\|f\|_{\mathcal{W}^{k, p}} & \text { for } 1 \leqslant p<\infty, \\
\left\|f^{\alpha}-f\right\|_{\mathcal{W}^{k, p}} \leqslant \frac{\max \left\{\left|\alpha_{i}\right| / a_{i}^{k}: i \in \mathbb{N}_{N-1}\right\}}{1-\max \left\{\left|\alpha_{i}\right| / a_{i}^{k}: i \in \mathbb{N}_{N-1}\right\}}\left\|I_{d}-L\right\|\|f\|_{\mathcal{W}^{k, p}} & \text { for } p=\infty
\end{array}
$$

Proof. The previous proposition in conjunction with the triangle inequality yields

$$
\begin{aligned}
\left\|f^{\alpha}-f\right\|_{\mathcal{W}^{k, p}} & \leqslant K\left\|f^{\alpha}-b\right\|_{\mathcal{W}^{k, p}} \\
& \leqslant K\left[\left\|f^{\alpha}-f\right\|_{\mathcal{W}^{k, p}}+\|f-L f\|_{\mathcal{W}^{k, p}}\right] \\
& \leqslant K\left[\left\|f^{\alpha}-f\right\|_{\mathcal{W}^{k, p}}+\left\|I_{d}-L\right\|\|f\|_{\mathcal{W}^{k, p}}\right]
\end{aligned}
$$

where

$$
K= \begin{cases}{\left[\sum_{i \in \mathbb{N}_{N-1}} \frac{\left|\alpha_{i}\right|^{p}}{a_{i}^{k p-1}}\right]^{1 / p}} & \text { for } p \in[1, \infty), \\ \max \left\{\frac{\left|\alpha_{i}\right|}{a_{i}^{k}}: i \in \mathbb{N}_{N-1}\right\} & \text { for } p=\infty,\end{cases}
$$

from which the result follows at once.

Theorem 4.3. For the scaling factors satisfying conditions prescribed in Theorem 3.4, the self-referential operator $\mathcal{F}^{\alpha}: \mathcal{W}^{k, p}(I) \rightarrow \mathcal{W}^{k, p}(I)$ is a bounded linear operator that reduces to the identity operator for $\alpha=0$.

Proof. Let $f_{1}$ and $f_{2}$ be in $\mathcal{W}^{k, p}(I)$ and let $\beta_{1}, \beta_{2}$ be reals. The functional equation for $f_{1}^{\alpha}=\mathcal{F}^{\alpha}\left(f_{1}\right)$ and $f_{2}^{\alpha}=\mathcal{F}^{\alpha}\left(f_{2}\right)$ are given by

$$
\begin{array}{ll}
f_{1}^{\alpha}(x)=f_{1}(x)+\alpha_{i}\left(f_{1}^{\alpha}-L f_{1}\right)\left(L_{i}^{-1}(x)\right), & x \in I_{i}, \quad i \in \mathbb{N}_{N-1}, \\
f_{2}^{\alpha}(x)=f_{2}(x)+\alpha_{i}\left(f_{2}^{\alpha}-L f_{2}\right)\left(L_{i}^{-1}(x)\right), & x \in I_{i}, i \in \mathbb{N}_{N-1} .
\end{array}
$$


Therefore,

$$
\left(\beta_{1} f_{1}^{\alpha}+\beta_{2} f_{2}^{\alpha}\right)(x)=\left(\beta_{1} f_{1}+\beta_{2} f_{2}\right)(x)+\alpha_{i}\left[\beta_{1} f_{1}^{\alpha}+\beta_{2} f_{2}^{\alpha}-L\left(\beta_{1} f_{1}+\beta_{2} f_{2}\right)\right]\left(L_{i}^{-1}(x)\right),
$$

from which it follows that $\beta_{1} f_{1}^{\alpha}+\beta_{2} f_{2}^{\alpha}$ is a fixed point of the operator

$$
(T g)(x)=\left(\beta_{1} f_{1}+\beta_{2} f_{2}\right)(x)+\alpha_{i}\left(g-L\left(\beta_{1} f_{1}+\beta_{2} f_{2}\right)\right)\left(L_{i}^{-1}(x)\right) .
$$

By the uniqueness of the fixed point we see that

$$
\mathcal{F}^{\alpha}\left(\beta_{1} f_{1}+\beta_{2} f_{2}\right)=\left(\beta_{1} f_{1}+\beta_{2} f_{2}\right)^{\alpha}=\beta_{1} f_{1}^{\alpha}+\beta_{2} f_{2}^{\alpha}=\beta_{1} \mathcal{F}^{\alpha}\left(f_{1}\right)+\beta_{2} \mathcal{F}^{\alpha}\left(f_{2}\right),
$$

proving the linearity of $\mathcal{F}^{\alpha}$. In view of the previous theorem we have

$$
\begin{aligned}
\left\|\mathcal{F}^{\alpha}(f)\right\|_{\mathcal{W}^{k, p}}=\left\|f^{\alpha}\right\|_{\mathcal{W}^{k, p}} & \leqslant\left\|f^{\alpha}-f\right\|_{\mathcal{W}^{k, p}}+\|f\|_{\mathcal{W}^{k, p}} \\
& \leqslant \frac{K}{1-K}\left\|I_{d}-L\right\|\|f\|_{\mathcal{W}^{k, p}}+\|f\|_{\mathcal{W}^{k, p}} \\
& =\left[1+\frac{K}{1-K}\left\|I_{d}-L\right\|\right]\|f\|_{\mathcal{W}^{k, p}},
\end{aligned}
$$

which ensures that $\mathcal{F}^{\alpha}$ is bounded and

$$
\left\|\mathcal{F}^{\alpha}\right\| \leqslant 1+\frac{K}{1-K}\left\|I_{d}-L\right\| .
$$

The last part of the theorem follows by noting that for $\alpha=0, f^{\alpha}=f$.

Theorem 4.4. Consider a scale vector $\alpha \in \mathbb{R}^{N-1}$ whose components satisfy

$$
\begin{gathered}
{\left[\sum_{i \in \mathbb{N}_{N-1}} \frac{\left|\alpha_{i}\right|^{p}}{a_{i}^{k p-1}}\right]^{1 / p}<\min \left\{1,\|L\|^{-1}\right\} \quad \text { for } p \in[1, \infty),} \\
\max _{i \in \mathbb{N}_{N-1}}\left\{\frac{\left|\alpha_{i}\right|}{a_{i}^{k}}\right\}<\min \left\{1,\|L\|^{-1}\right\} \quad \text { for } p=\infty .
\end{gathered}
$$

The corresponding fractal operator $\mathcal{F}^{\alpha}$ is bounded below. In particular, $\mathcal{F}^{\alpha}$ is injective and has a closed range. In fact, $\mathcal{F}^{\alpha}: \mathcal{W}^{k, p}(I) \rightarrow \mathcal{F}^{\alpha}\left(\mathcal{W}^{k, p}(I)\right)$ is a topological isomorphism.

Proof. From Proposition 4.1,

$\|f\|_{\mathcal{W}^{k, p}}-\left\|f^{\alpha}\right\|_{\mathcal{W}^{k, p}} \leqslant\left\|f-f^{\alpha}\right\|_{\mathcal{W}^{k, p}} \leqslant K\left\|f^{\alpha}-L f\right\|_{\mathcal{W}^{k, p}} \leqslant K\left[\left\|f^{\alpha}\right\|_{\mathcal{W}^{k, p}}+\|L\|\|f\|_{\mathcal{W}^{k, p}}\right]$,

where $K$ is as prescribed in Theorem 4.2. Also, by the stated assumption on the scale vector, we have $K<\|L\|^{-1}$. Thus

$$
\|f\|_{\mathcal{W}^{k, p}} \leqslant \frac{1+K}{1-K\|L\|}\left\|f^{\alpha}\right\|_{\mathcal{W}^{k, p}}
$$

That is, the operator $\mathcal{F}^{\alpha}$ is bounded below and hence, in particular, it is injective. To

'importantly'? 
prove that $\mathcal{F}^{\alpha}\left(\mathcal{W}^{k, p}(I)\right)$ is closed, let $f_{n}^{\alpha}$ be a sequence in $\mathcal{F}^{\alpha}\left(\mathcal{W}^{k, p}(I)\right)$ such that $f_{n}^{\alpha} \rightarrow g$. In particular, $\left\{f_{n}^{\alpha}=\mathcal{F}^{\alpha}\left(f_{n}\right)\right\}$ is a Cauchy sequence in $\mathcal{F}^{\alpha}\left(\mathcal{W}^{k, p}(I)\right)$. Since

$$
\left\|f_{n}-f_{m}\right\|_{\mathcal{W}^{k, p}} \leqslant \frac{1+K}{1-K\|L\|}\left\|f_{n}^{\alpha}-f_{m}^{\alpha}\right\|_{\mathcal{W}^{k, p}}
$$

it follows that $\left\{f_{n}\right\}$ is a Cauchy sequence in $\mathcal{W}^{k, p}(I)$. Since $\mathcal{W}^{k, p}(I)$ is a complete space, there exists an $f \in \mathcal{W}^{k, p}(I)$ such that $f_{n} \rightarrow f$, and, consequently, by the continuity of the fractal operator $\mathcal{F}^{\alpha}$ we conclude that $g=\mathcal{F}^{\alpha}(f)=f^{\alpha}$. From the bounded inverse theorem (see, for example, [5]) it now follows that the inverse of the map $\mathcal{F}^{\alpha}: \mathcal{W}^{k, p}(I) \rightarrow \mathcal{F}^{\alpha}\left(\mathcal{W}^{k, p}(I)\right)$ is a bounded linear operator, completing the proof.

Theorem 4.5. For a scale vector $\alpha \in \mathbb{R}^{N-1}$ whose components satisfy

$$
\begin{gathered}
{\left[\sum_{i \in \mathbb{N}_{N-1}} \frac{\left|\alpha_{i}\right|^{p}}{a_{i}^{k p-1}}\right]^{1 / p}<\left(1+\left\|I_{d}-L\right\|\right)^{-1} \quad \text { for } p \in[1, \infty),} \\
\max _{i \in \mathbb{N}_{N-1}}\left\{\frac{\left|\alpha_{i}\right|}{a_{i}^{k}}\right\}<\left(1+\left\|I_{d}-L\right\|\right)^{-1} \quad \text { for } p=\infty,
\end{gathered}
$$

the fractal operator $\mathcal{F}^{\alpha}$ is a topological automorphism on $\mathcal{W}^{k, p}(I)$. Furthermore, with $K$ as in Theorem 4.2,

$$
\frac{1-K\|L\|}{1+K}\|f\|_{\mathcal{W}^{k, p}} \leqslant\left\|\mathcal{F}^{\alpha}(f)\right\|_{\mathcal{W}^{k, p}} \leqslant 1+\frac{K}{1-K}\left\|I_{d}-L\right\|\|f\|_{\mathcal{W}^{k, p}} .
$$

Proof. From Theorem 4.2 it can be easily seen that

$$
\left\|I_{d}-\mathcal{F}^{\alpha}\right\| \leqslant \frac{K}{1-K}\left\|I_{d}-L\right\| .
$$

In view of the assumption on the scaling vector $\alpha$, we get $K<\left(1+\left\|I_{d}-L\right\|\right)^{-1}$ and hence $\left\|I_{d}-\mathcal{F}^{\alpha}\right\|<1$. Consequently, the Neumann series $\sum_{j=0}^{\infty}\left(I_{d}-\mathcal{F}^{\alpha}\right)^{j}$ is convergent in the operator norm and $\mathcal{F}^{\alpha}=I_{d}-\left(I_{d}-\mathcal{F}^{\alpha}\right)$ is invertible (see, for example, [5]). Bearing in mind that

$$
\|L\|=\left\|I_{d}-\left(I_{d}-L\right)\right\| \leqslant 1+\left\|I_{d}-L\right\|,
$$

the bounds on $\left\|\mathcal{F}^{\alpha}(f)\right\|_{\mathcal{W}^{k, p}}$ follow from Theorem 4.3 and 4.4 .

The existence of Schauder bases for Sobolev spaces is desired for demonstrating the existence of solutions of nonlinear boundary-value problems. Sometimes it is interesting to look for the global structure involved in a given problem, and self-referentiality may therefore be advantageous. Hence, it is worth searching for a Schauder basis for $\mathcal{W}^{k, p}(I)$ consisting of fractal functions. It is to this that we now turn. First let us recall the following theorem, which asserts the existence of Schauder basis for $\mathcal{W}^{k, p}(I)$, where $p \geqslant 1$ is a real number. We briefly reproduce its proof here for the sake of completeness. Without

'helpful if one wants to prove the existence...'?

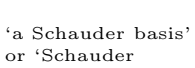
or 'Schauder bases'? loss of generality assume that $I=[0,1]$. 
Theorem 4.6 (Fučík [8, Theorem 4.7]). For $1 \leqslant p<\infty, \mathcal{W}^{k, p}(I)$ has a Schauder basis.

Proof. Proof is by induction with respect to $k$. For $k=0, \mathcal{W}^{0, p}(I)=\mathcal{L}^{p}(I)$, and it has Word added - OK? a Schauder basis. Let $\left\{f_{n}^{k}\right\}$ be a Schauder basis for $\mathcal{W}^{k, p}(I)$ and let $\left\{\beta_{n}^{k}\right\}$ be a sequence of continuous linear functionals such that for each $f \in \mathcal{W}^{k, p}(I), f=\sum_{n=1}^{\infty} \beta_{n}^{k}(f) f_{n}^{k}$. For $f \in \mathcal{W}^{k, p}(I)$, set

$$
\begin{aligned}
f_{1}^{k+1}(x) & \equiv 1, & \beta_{1}^{k+1}(f) & =f(0), \\
f_{n}^{k+1}(x) & =\int_{0}^{x} f_{n-1}^{k}(t) \mathrm{d} t, & \beta_{n}^{k+1}(f) & =\beta_{n-1}^{k}\left(f^{\prime}\right), \quad n \geqslant 2 .
\end{aligned}
$$

Then $\left\{f_{n}^{k+1}\right\}$ is a Schauder basis in $\mathcal{W}^{k+1, p}(I)$.

Theorem 4.7. For $1 \leqslant p<\infty$, the space $\mathcal{W}^{k, p}(I)$ admits a Schauder basis consisting of self-referential functions.

Proof. Let $\left\{f_{n}\right\}$ be a Schauder basis for $\mathcal{W}^{k, p}(I)$ with associated coefficient functionals $\left\{\beta_{n}\right\}$. Consider the scaling factors $\alpha_{i}, i \in \mathbb{N}_{N-1}$, such that the condition prescribed in Theorem 4.5 is satisfied so that $\mathcal{F}^{\alpha}$ is a topological automorphism on $\mathcal{W}^{k, p}(I)$. Let $f \in \mathcal{W}^{k, p}(I)$. Then $\left(\mathcal{F}^{\alpha}\right)^{-1}(f) \in \mathcal{W}^{k, p}(I)$ and

$$
\left(\mathcal{F}^{\alpha}\right)^{-1}(f)=\sum_{n=1}^{\infty} \beta_{n}\left(\left(\mathcal{F}^{\alpha}\right)^{-1}(f)\right) f_{n}
$$

Since $\mathcal{F}^{\alpha}$ is a bounded linear map we obtain

$$
f=\sum_{n=1}^{\infty} \beta_{n}\left(\left(\mathcal{F}^{\alpha}\right)^{-1}(f)\right) f_{n}^{\alpha}
$$

Next we prove that the representation is unique. Let $f=\sum_{n=1}^{\infty} \gamma_{n} f_{n}^{\alpha}$ be another representation of $f$. Continuity of $\left(\mathcal{F}^{\alpha}\right)^{-1}$ ensures that

$$
\left(\mathcal{F}^{\alpha}\right)^{-1}(f)=\sum_{n=1}^{\infty} \gamma_{n} f_{n}
$$

and hence

$$
\gamma_{n}=\beta_{n}\left(\left(\mathcal{F}^{\alpha}\right)^{-1}(f)\right), \quad n \in \mathbb{N} .
$$

Thus $\left\{f_{n}^{\alpha}\right\}$ is a Schauder basis for $\mathcal{W}^{k, p}(I)$ comprising of self-referential functions.

For $p=2$, the Sobolev space $\mathcal{W}^{k, 2}(I)=\mathcal{H}^{k}(I)$ is a Hilbert space and hence we can talk about the adjoint (in the usual sense) $\left(\mathcal{F}^{\alpha}\right)^{*}$ of the fractal operator $\mathcal{F}^{\alpha}$. We have the following. 
Theorem 4.8. For a scale vector $\alpha \in \mathbb{R}^{N-1}$ satisfying

$$
\left[\sum_{i \in \mathbb{N}_{N-1}} \frac{\left|\alpha_{i}\right|^{2}}{a_{i}^{2 k-1}}\right]^{1 / 2}<\min \left\{1,\|L\|^{-1}\right\},
$$

we have

$$
\mathcal{H}^{k}(I)=\operatorname{rg}\left(\mathcal{F}^{\alpha}\right) \oplus \operatorname{ker}\left(\left(\mathcal{F}^{\alpha}\right)^{*}\right),
$$

where $\operatorname{rg}(A)$ and $\operatorname{ker}(A)$ represent the range and kernel of an operator $A$. Also, $\left(\mathcal{F}^{\alpha}\right)^{*}$ is surjective.

Proof. From the proof of Theorem 4.4 it follows that for a scale vector $\alpha$ satisfying the stated hypothesis, $\operatorname{rg}\left(\mathcal{F}^{\alpha}\right)$ is closed. By the orthogonal decomposition theorem for a Hilbert space (see, for example, [5]),

$$
\mathcal{H}^{k}(I)=\operatorname{rg}\left(\mathcal{F}^{\alpha}\right) \oplus \operatorname{rg}\left(\mathcal{F}^{\alpha}\right)^{\perp}=\operatorname{rg}\left(\mathcal{F}^{\alpha}\right) \oplus \operatorname{ker}\left(\left(\mathcal{F}^{\alpha}\right)^{*}\right) .
$$

Again by the orthogonal decomposition,

$$
\mathcal{H}^{k}(I)=\overline{\operatorname{rg}\left(\left(\mathcal{F}^{\alpha}\right)^{*}\right)} \oplus \overline{\operatorname{rg}\left(\left(\mathcal{F}^{\alpha}\right)^{*}\right)}{ }^{\perp}=\overline{\operatorname{rg}\left(\left(\mathcal{F}^{\alpha}\right)^{*}\right)} \oplus \operatorname{rg}\left(\left(\mathcal{F}^{\alpha}\right)^{*}\right)^{\perp}=\overline{\operatorname{rg}\left(\left(\mathcal{F}^{\alpha}\right)^{*}\right)} \oplus \operatorname{ker}\left(\mathcal{F}^{\alpha}\right) .
$$

For a scale vector satisfying the given condition, $\mathcal{F}^{\alpha}$ is injective. That is, $\operatorname{ker}\left(\mathcal{F}^{\alpha}\right)=\{0\}$. Consequently, $\mathcal{H}^{k}(I)=\overline{\operatorname{rg}\left(\left(\mathcal{F}^{\alpha}\right)^{*}\right)}$, i.e. $\operatorname{rg}\left(\left(\mathcal{F}^{\alpha}\right)^{*}\right)$ is dense in $\mathcal{H}^{k}(I)$. For a linear and bounded operator of a Hilbert space, its range is closed if and only if the range of its adjoint is closed (see, for example, [5]). Therefore, $\operatorname{rg}\left(\left(\mathcal{F}^{\alpha}\right)^{*}\right)$ is closed and hence $\operatorname{rg}\left(\left(\mathcal{F}^{\alpha}\right)^{*}\right)=\mathcal{H}^{k}(I)$.

Theorem 4.9. For a scale vector $\alpha$ satisfying

$$
\left[\sum_{i \in \mathbb{N}_{N-1}} \frac{\left|\alpha_{i}\right|^{2}}{a_{i}^{2 k-1}}\right]^{1 / 2}<\left(1+\left\|I_{d}-L\right\|\right)^{-1},
$$

the fractal operator $\mathcal{F}^{\alpha}: \mathcal{H}^{k}(I) \rightarrow \mathcal{H}^{k}(I)$ is Fredholm with index zero.

Proof. With the stated assumption on $\alpha$, Theorem 4.5 asserts that the operator $\mathcal{F}^{\alpha}$ is an isomorphism. Therefore, by the following theorem (see [19]) $\mathcal{F}^{\alpha}$ is Fredholm.

A linear bounded operator $A$ is Fredholm if and only if $A=B+F$, where $B$ is an isomorphism and $F$ has finite rank.

Also,

$$
\operatorname{ind} \mathcal{F}^{\alpha}:=\operatorname{dim} \operatorname{ker}\left(\mathcal{F}^{\alpha}\right)-\operatorname{codim} \operatorname{rg}\left(\mathcal{F}^{\alpha}\right)=0,
$$

delivering the proof.

Remark 4.10. Throughout this paper we have confined our discussion to real-valued functions. However, we remark that many of our results apply immediately to complex- 
valued functions defined on a real compact interval. For instance, denoting the real and imaginary parts of $f$ by $f_{\text {re }}$ and $f_{\mathrm{im}}$, respectively, one may consider the operator

$$
\mathcal{F}_{C}^{\alpha}: \mathcal{W}^{k, p}(I, \mathbb{C}) \rightarrow \mathcal{W}^{k, p}(I, \mathbb{C}), \quad \mathcal{F}_{C}^{\alpha}(f)=\mathcal{F}_{C}^{\alpha}\left(f_{\text {re }}+\mathrm{i} f_{\text {im }}\right)=\mathcal{F}^{\alpha}\left(f_{\mathrm{re}}\right)+\mathrm{i} \mathcal{F}^{\alpha}\left(f_{\text {im }}\right) .
$$

It is not hard to show that $\mathcal{F}_{C}^{\alpha}$ is a bounded linear operator. Other properties of $\mathcal{F}_{C}^{\alpha}$ can be dealt with similarly.

Acknowledgements. The authors gratefully and sincerely thank the anonymous referee for his/her very valuable comments and constructive criticism. Furthermore, we warmly thank the referee for providing some good additional references.

\section{References}

1. M. F. Barnsley, Fractal functions and interpolation, Constr. Approx. 2 (1986), 303-329.

2. M. F. BARnsley And A. N. Harrington, The calculus of fractal interpolation functions, J. Approx. Theory 57(1) (1989), 14-34.

3. M. F. BARnsley And P. Massopust, Bilinear fractal interpolation and box dimension, J. Approx. Theory 192 (2015), 362-378.

4. M. F. Barnsley, M. Hegland and P. Massopust, Numerics and fractals, Bull. Inst. Math. Acad. Sin. 9(3) (2014), 389-430.

5. J. B. Conway, A course in functional analysis, 2nd edn (Springer, 1996).

6. L. C. Evans, Partial differential equations, 1st edn (American Mathematical Society, Providence, RI, 1998).

7. Z. G. Feng, Y. Z. Feng And Z. Y. YuAn, Fractal interpolation surfaces with function vertical scaling factors, Appl. Math. Lett. 25(11) (2012), 1896-1900.

8. S. FUČík, Fredholm alternative for nonlinear operators in Banach spaces and its applications to differential and integral equations, Čas Pro Pěst. Mat. 96 (1971), 371-390.

9. P. Massopust, Fractal functions, fractal surfaces, and wavelets (Academic Press, 1995).

10. P. Massopust, Fractal functions and their applications. Chaos, Solitons \& Fractals 8(2) (1997), 171-190.

11. P. Massopust, Fractal functions, splines, and Besov and Triebel-Lizorkin spaces, in Fractals in engineering: new trends and applications, ed. J. Lévy-Véhel and E. Lutton, pp. 21-32 (Springer, 2005).

12. P. MAssopust, Local fractal functions and function spaces, Springer Proceedings in Mathematics \& Statistics: Fractals, Wavelets, and Their Applications, Volume 92, pp. 245-270 (Springer, 2014).

13. P. Massopust, On local fractal functions in Banach spaces, AIP Conf. Proc. 1648 (2015), 640002-01-640002-04.

14. M. A. NAvascuÉs, Fractal polynomial interpolation, Z. Analysis Anwend. 25(2) (2005), 401-418.

15. M. A. NAvascuÉs, Fractal approximation, Complex Analysis Operat. Theory 4(4) (2010), 953-974.

16. M. A. Navascués, Fractal Haar sytem, Nonlin. Analysis TMA 74 (2011), 4152-4165.

17. M. A. NavascuÉs, Fractal bases of $L_{p}$ spaces, Fractals 20 (2012), 141-148.

18. M. A. NAvAscuÉs And M. V. Sebastián, Smooth fractal interpolation, J. Inequal. Not cited in text! Applicat. 2006 (2006), 78734.

19. A. G. Ramm, A simple proof of the Fredholm alternative and a characterization of the Fredholm operators, Am. Math. Mon. 108(9) (2001), 855-860.

20. H. Triebel, Theory of function spaces (Birkhäuser, 1983). 
21. H. TRIEBEL, Theory of function spaces II (Birkhäuser, 1992).

22. P. Viswanathan, A. K. B. Chand And M. A. Navascués, Fractal perturbation preserving fundamental shapes: bounds on the scale factors, J. Math. Analysis Applic. 419 (2014), 804-817.

23. P. Viswanathan, M. A. Navascués and A. K. B. Chand, Fractal polynomials and maps in approximation of continuous functions, Numer. Func. Analysis Optim. 37(1) (2015), http://doi.org/brvp.

24. P. Viswanathan, M. A. Navascués and A. K. B. Chand, Associate fractal functions in $\mathcal{L}^{p}$-spaces and in one-sided uniform approximation, J. Math. Analysis Applic. 433(2) (2015), 862-876.

25. H. Y. WANG AND J. S. YU, Fractal interpolation functions with variable parameters and their analytical properties, J. Approx. Theory 175 (2013), 1-18. 